ORIGINAL ARTICLE

\title{
Histopathological patterns of melanoma metastases in sentinel lymph nodes
}

\author{
C A Murray, W L Leong, D R McCready, D M Ghazarian
}

See end of article for authors' affiliations

.....................

Correspondence to:

Dr D M Ghazarian,

Department of Pathology,

Princess Margaret

Hospital, University Health

Network, Toronto, M5G

2M9 Ontario, Canada;

danny.ghazarian@

uhn.on.ca

Accepted for publication 6 June 2003

\begin{abstract}
Aims: Sentinel lymph node biopsy (SLNB) is an important component in the staging and treatment of cutaneous melanoma (CM). The medical literature provides only limited information regarding melanoma sentinel lymph node (SLN) histology. This report details the specific histological patterns of melanoma metastases in sentinel lymph nodes (SLNs) and highlights some key factors in evaluating SLNs for melanoma.

Methods: From 281 SLNB cases between June 1998 and May 2002, 79 consecutive cases of SLN biopsies positive for metastases from CM were retrospectively reviewed. The important characteristics of the SLNs and the metastatic foci are described.

Results: The median size of positive SLNs was $17 \mathrm{~mm}$ (range, 5-38). SLNs had a median of two metastatic foci (range, 1-11), with the largest foci being a median of $1.1 \mathrm{~mm}$ in size (range, 0.05-24). S-100 and HMB-45 staining was positive in $100 \%$ and $92 \%$ of the detected metastatic foci, respectively. The metastatic melanoma cells were epithelioid, spindled, and mixed in $86 \%, 5 \%$, and $9 \%$ of cases. Metastatic foci were most often (86\%) found in the subcapsular region of the SLN. Benign naevic cells were found coexisting in $14 \%$ of positive SLNs.

Conclusions: Staining for S100 is more sensitive than HMB-45 (100\% v92\%), but HMB-45 staining helped to distinguish benign naevic cells from melanoma. The subcapsular region was crucial in SLN evaluation, because it contained the metastases in $86 \%$ of cases. Evaluation of the subcapsular space should not be compromised by cautery artefacts or incomplete excision of the SLN.
\end{abstract}

"There is now convincing evidence that sentinel lymph nodes are accurate predictors of the status of the entire nodal basin"

The methodology of using a vital blue dye to detect and excise the first draining (sentinel) lymph node(s) in melanoma was described by Morton et al in $1992 .^{5}$ The technique was later modified by the addition of radiocolloid. ${ }^{6}$ There is now convincing evidence that sentinel lymph nodes (SLNs) are accurate predictors of the status of the entire nodal basin. ${ }^{5}$ If detailed examination of the SLNs is negative for malignancy, then the chance of disease being present in the other lymph nodes in the same nodal basin is estimated to be less than $2 \%$ (negative predictive value 98.6\%). ${ }^{7}$ Conversely, if the SLNs are involved with metastatic cells, other nodes have an approximately $20 \%$ chance of harbouring metastasis, for which a completion lymphadenectomy is recommended. ${ }^{7}$ In general, the prognosis is worse the higher the number of lymph nodes involved, ${ }^{8}$ and adjuvant treatments such as interferon are often used. However, patient outcomes are not entirely predictable. ${ }^{9}$ Ten year mortality with nodal involvement ranges from $37 \%$ to $85 \%{ }^{8}$

The morphology of the melanoma cells within the SLNs has never been classified, prompting us to detail the results of our experience. This publication presents data from over four years of sentinel lymph node biopsy (SLNB) collection. The histological characteristics are described, and the possible future applications relating to the correlation of these features with clinical outcomes will be discussed.

\section{METHODS AND MATERIALS}

Our study was conducted at Princess Margaret Hospital, University Health Network, at the University of Toronto, Canada. Our institution began standardised synoptic reporting of melanoma cases in 1998. Between June 1998 and May 2002, the indications for SLNB were primary tumours with thickness greater than $1.0 \mathrm{~mm}$, or with the presence of ulceration. Patients with clinically involved regional lymph node and systemic metastasis were excluded.

\section{SLNBS}

SLNBs were performed according to McCready et al. ${ }^{10}$ Briefly, peritumour intradermal injections of unfiltered technitium99 sulfa colloid $(20-40 \mathrm{mBq}$ in $0.5 \mathrm{ml})$, with or without patent blue dye $(0.5 \mathrm{ml})$, were used to identify SLNs intraoperatively with a $\gamma$ probe. All hot and/or blue SLNs were excised through a small skin incision until the background count was less than $10 \%$ of the hottest node.

\footnotetext{
Abbreviations: $C M$, cutaneous melanoma; $H \& E$, haematoxylin and eosin; IHC, immunohistochemistry; SLN, sentinel lymph node; SLNB, sentinel lymph node biopsy
} 


\section{Pathology}

The pathology assessments were done according to Cochran. ${ }^{11}$ In brief, the lymph nodes were either bivalved or cut into $3 \mathrm{~mm}$ blocks, depending on the size of the node. Sections 1,3 , and 5 were stained with haematoxylin and eosin (H\&E); sections 2 and 4 were immunohistochemically stained for S-100 (polyclonal antibody; Dako; 1/3000 dilution) and HMB-45 (monoclonal antibody; Dako; 1/50 dilution).

\section{Data collection and analyses}

Data relating to the primary tumour histopathological characteristics were prospectively recorded in a synoptic report. All the SLN specimens were analysed by the same dermatopathologist (DG).

\section{RESULTS}

In total, there were 281 SLNB cases for melanoma between June 1998 and May 2002. The success rate of identifying at least one SLN was $100 \%$, with a median of two SLNs (range, $1-8)$ removed for each patient. Seventy nine of the 281 cases $(28 \%)$ were positive for nodal metastasis. Of the SLN positive patients, $62 \%$ were men, the average age was 50 years, the median thickness of the primary tumour was $2.65 \mathrm{~mm}$ (range, 1.0-11), and 19\% were ulcerated. Figure l shows the sites of the primary tumours. Wide local excisions were performed before SLNB in $39 \%$ of the patients, and at the same time as SLN excision in the remaining $61 \%$. Of the 79 SLN positive patients, a total of 215 SLNs were assessed, of which $46 \%$ were positive (median, one/patient; range, 1-3). All of the metastatic foci were positive for S-100, but only $92 \%$ were positive for HMB-45. The median size of the SLNs measured was $17 \mathrm{~mm}$ (range, 5-38 mm). The median number of foci observed was two (range, 1-11). The median size of the largest focus in each case was $1.1 \mathrm{~mm}$ (range, $0.05-24)$. The metastatic cells in the SLNs were epithelioid in $86 \%$ cases, compared with 5\% spindled and 9\% mixed epithelioid and spindled type (fig 2). Thirty two per cent of the malignant foci had a subcapsular location, 44\% a subcapsular and parenchymal location, 11\% a parenchymal location, $5 \%$ a sinusoidal and subcapsular location, but none a sinusoidal location alone (fig 3 ). Benign naevic cells were found coexisting in 11 of the 79 positive SLN cases. Extracapsular spread was noted in four cases.

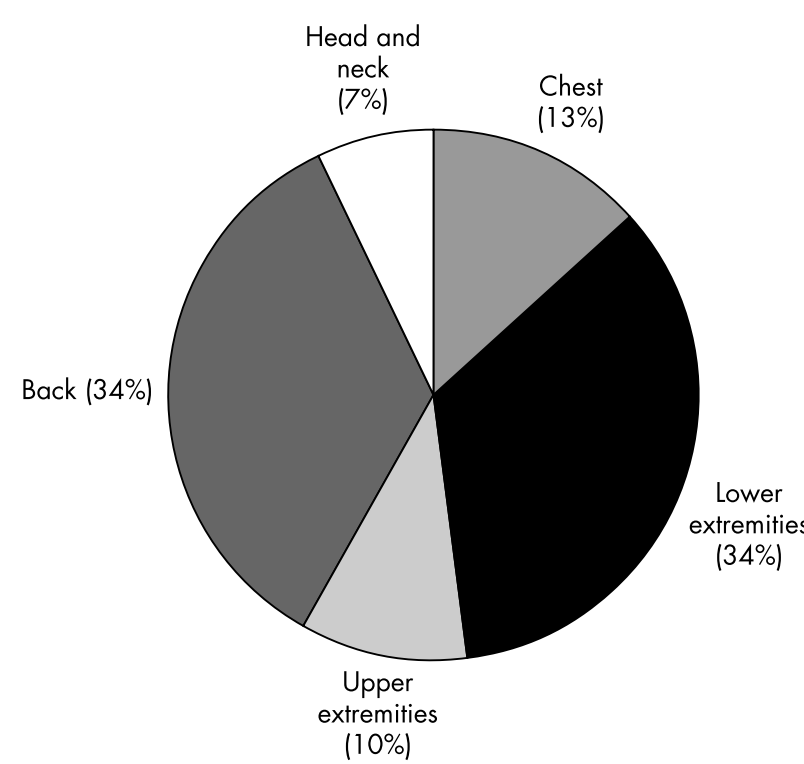

Figure 1 Pie chart illustrating the location of the primary melanomas in patients with positive sentinel lymph node biopsies.

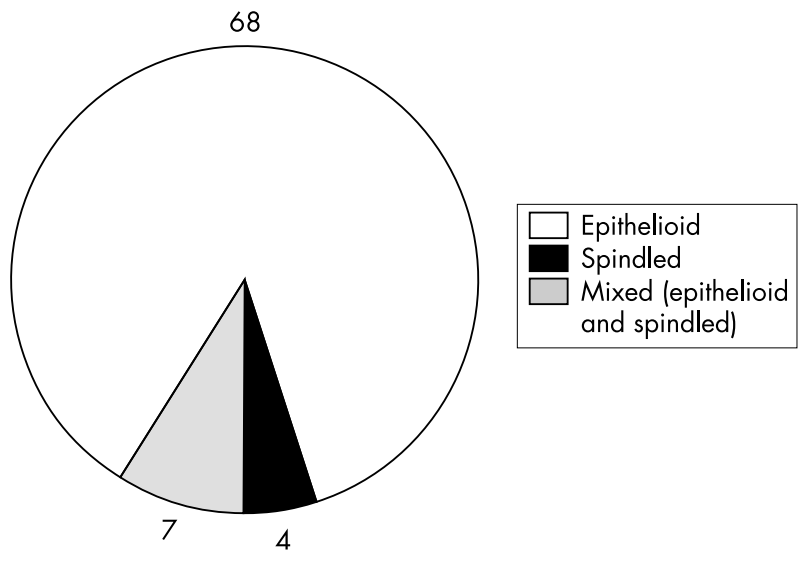

Figure 2 Pie chart illustrating the frequency of different metastatic melanoma cell types, as recognised by haematoxylin and eosin staining. Metastatic cells were solely epithelioid in morphology in $86 \%$ (68 of 79) of cases.

\section{DISCUSSION}

The management of melanoma is complex and is dependent on the staging at the initial diagnosis. For patients with intermediate to thick CM, SLNB is the standard nodal staging procedure. SLNB is very accurate in predicting the status of the remaining regional lymph nodes, ${ }^{72-14}$ and is currently the most significant independent prognostic indicator for survival when compared with all other factors, including tumour thickness and the presence of ulceration. ${ }^{75-17}$ The importance of SLNB in the management of melanoma is reflected in the most recent AJCC staging system. ${ }^{8}$ World Health Organisation representatives have recently stated that SLNB is the standard of care in the management of patients with melanoma and is fundamental to further directives in surgery and adjuvant treatment strategies. ${ }^{15}$ Several studies, including the multicentre selective lymphadenectomy trial, ${ }^{14}$ will help to answer the question of whether or not SLNB offers any survival advantage in these patients.

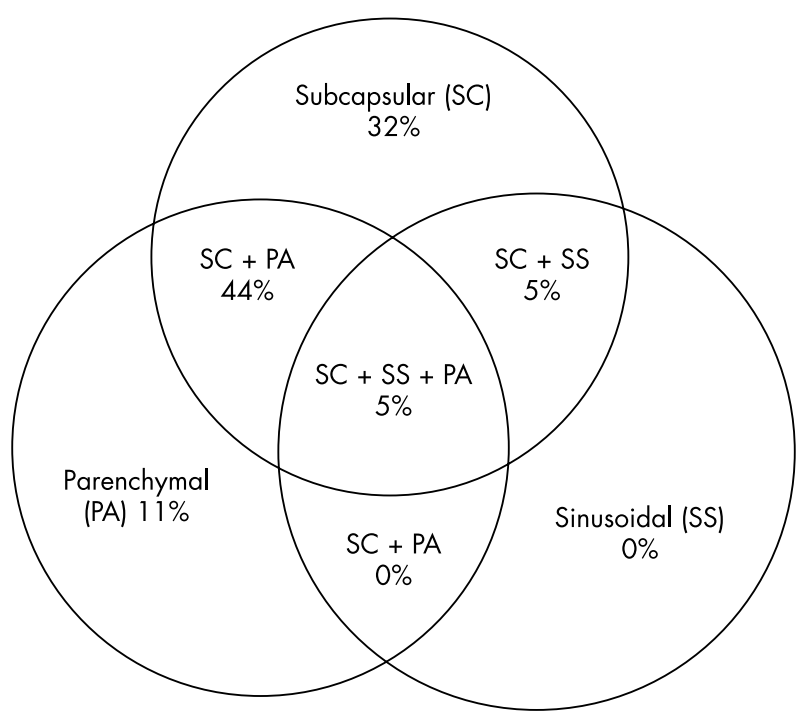

Figure 3 Distribution of metastatic foci within the sentinel lymph node, as identified by haematoxylin and eosin and immunohistochemical techniques. Malignant foci were frequently detected in more than one nodal sublocation. The subcapsular space was involved in $86 \%$ (68 of 79) of sentinel lymph nodes examined. 
The current standard for the diagnosis of SLN metastasis is based on routine H\&E histology and immunohistochemistry (IHC). The sensitivity of detection is increased with IHC, multiple sectioning, ${ }^{13}{ }^{18}$ and reverse transcriptase polymerase chain reaction (RT-PCR) techniques. ${ }^{5}{ }^{17-23}$ In one series, the relapse rate over 28 months for $\mathrm{H} \& \mathrm{E}$ detected SLN positive patients was $67 \%$, whereas the relapse rate for SLN negative patients was $2-6 \%$ during the same period. ${ }^{17}$ Patients who were RT-PCR positive but histologically negative relapsed 13$20 \%$ of the time. ${ }^{24}$ However, the role of such molecular genetic techniques in identifying melanoma proteins remains undefined, despite greater sensitivity. ${ }^{25}$ False positives resulting from benign naevic cells raise concern and necessitate further refinement. Attempts to develop a radiological test, such as positron emission tomography, to identify malignant spread within the lymphatics have been disappointing. ${ }^{26}$

In patients with breast cancer, an attempt was made to define pathological features that portend a more specific prognosis. Chu et al noted that non-sentinel nodes were never involved with breast metastases if the SLN micrometastatic focus was less than $2 \mathrm{~mm}$ in size, and detected only by IHC. ${ }^{27}$ Czerniecki's group similarly found an absence of spread beyond the SLN if micrometastatic foci were less than $2 \mathrm{~mm}$ and the primary breast tumour was smaller than $2 \mathrm{~cm} .{ }^{28}$

In melanoma, Wagner et al failed to correlate the number of metastatic foci in SLNs with the risk of non-SLN involvement, but the study did not record the size of the foci. ${ }^{29}$ The Cavalieri principle has been used to estimate the tumour volume in primary and lymphatic melanoma. However, this technique is cumbersome and does not account for many other features of nodal histology. ${ }^{30}$ Other authors ${ }^{25}{ }^{31}$ have used a micrometric approach to approximate volumes. Starz et al reported a predictive model of outcome using a combination of the number of histological $1 \mathrm{~mm}$ sections involved with tumour and the centripetal depth of spread within the SLN. ${ }^{25}$ A multivariate Cox analysis showed that centripetal invasion within the SNL was an independent risk factor for distant metastasis and survival.

\section{"Surgeons should be aware that the subcapsular region is crucial in sentinel lymph node (SLN) evaluation and the architecture of the SLN can be disrupted easily if the procedure is not carried out with care"}

There is little literature on the histopathological features of the metastatic foci in SLNs for melanoma. Our goal is to describe these features so that they may be evaluated as potential predictive factors for future studies. We found that $86 \%$ of SLN positive cases involved the subcapsular region, so that this should be the primary location when examining sentinel nodes. However, $11 \%$ were only found when the parenchyma of the lymph node was examined. Surgeons should be aware that the subcapsular region is crucial in SLN evaluation and the architecture of the SLN can be disrupted easily if the procedure is not carried out with care. To reduce the false negative rate, surgeons should avoid crushing and excessive cautery usage to preserve the integrity of the SLN. It is also important not to cut into the SLN and complete excision of the whole SLN is crucial. Five percent of the SLN metastases demonstrated solely a spindle cell morphology; in primary CM, this morphology is thought to be associated with a better prognosis than epithelioid morphology. ${ }^{32} 33$

It is important to distinguish naevic cells from melanoma metastasis. In our experience, naevic cells are usually present within the capsule (intracapsular) or within the trabeculae, and typically stain negatively, or only faintly positively, with HMB-45. Reactive dendritic cells, nerve fragments, and benign naevic cells may each stain positively for S100.

\section{Take home messages}

- When examining sentinel lymph node biopsies for melanoma metastases we found that the S100 stain was $100 \%$ sensitive, whereas the HMB-45 stain allowed us to differentiate benign naevic cells from their malignant counterparts

- Examination at the subcapsular level, combined with the use of S100 staining, is the most practical and sensitive method to ensure the detection of micrometastatic nodal disease

- Evaluation of the subcapsular space should not be compromised by cautery artefacts or incomplete excision of the sentinel lymph node

Plasma cells, mast cells, and some calcifications within lymph nodes may infrequently stain with HMB-45. Some spindled melanoma cells may stain negatively with HMB-45. Malignant foci must be carefully searched for on each section, because small foci may only be seen on a single section and not confirmed on deeper cuts. ${ }^{11}$

In our study, all melanoma metastases in SLNs were detected by routine H\&E histology and S100 stains. MART-1 (melan-A), now used routinely as a melanoma marker in the assessment of SLN, was not used during the study period in our retrospective analysis. However, similar to S-100, MART1 cannot differentiate between benign naevic cells and melanoma. The HMB-45 immunostain was helpful in distinguishing benign naevic cells, which are usually HMB45 negative, from melanoma.

Of interest, it has recently been shown that a larger melanoma tumour burden in the SLNB has prognostic significance. ${ }^{34}$ Ranieri et al reported that both a greater total lymph node tumour volume and larger tumour nodules within the SLN were associated with worse outcomes. ${ }^{34}$ Correlation of size of the tumour deposit, number of foci, location of foci, and the cell type (epithelioid, spindled, or mixed) with other clinicopathological prognostic indicators is ongoing at our centre and will be reported separately.

\section{CONCLUSION}

Although SLNB has become the standard nodal assessment method for the management of CM, we hope to improve upon the current diagnostic accuracy by providing a detailed description of the histopathological features of the metastatic pattern in SLNs. We have found that the subcapsular site is the most common location for the metastatic deposits and that most metastatic cells have an epithelioid morphology. The S100 stain was $100 \%$ sensitive in our series, but the HMB-45 stain allowed us to differentiate benign naevic cells from their malignant counterparts. Examination at the subcapsular level, combined with the use of S100 staining, is the most practical and sensitive method to ensure the detection of micrometastatic nodal disease. To reduce the false negative rate, surgeons and pathologists who perform SLNB should be aware of the clinical and pathological pitfalls of SLNB, and must ensure the integrity of the SLN architecture during the procedure.

\section{Authors' affiliations}

C A Murray, Department of Dermatology, University of Toronto, Toronto, Ontario, Canada

D R McCready, W L Leong, Department of Surgical Oncology, Princess Margaret Hospital, Toronto M5G 2M9, Ontario, Canada 
D M Ghazarian, Department of Pathology, Princess Margaret Hospital, University Health Network, Toronto, Ontario, Canada

\section{REFERENCES}

1 Jemal A, Thomas A, Murray T, et al. Cancer statistics, 2002. CA Cancer J Clin 2002;52:23-47.

2 Greenlee RT, Hill-Harmon MB, Murray T, et al. Cancer statistics, 2001. CA Cancer J Clin 2001;5:15-36,

3 Chang AE, Karnell LH, Menck HR. The national cancer database report on cutaneous and non-cutaneous melanoma: a summary of 84,836 cases from the past decade. Cancer 1998;8:1664-78.

4 Rigel DS, Friedman RJ, Kopf AW. The incidence of malignant melanoma in the United States: issues as we approach the 21 st century. J Am Acad Dermatol 1996:34(5 pt1):839-47.

5 Morton DL, Wen DR, Wong JH, et al. Technical details of intraoperative lymphatic mapping for early stage melanoma. Arch Surg 1992;127:392-9.

6 Van der Veen H, Hoekstra OS, Paul MA, et al. Gamma probe-guided sentinel node biopsy to select patients with melanoma for lymphadenectomy. $\mathrm{Br} J$ Surg 1994:81:1769-70.

7 Gershenwald JE, Thompson W, Mansfield PF, et al. Multi-institutional melanoma lymphatic mapping experience: the prognostic value of sentinel node status in 612 stage I or II melanoma patients. J Clin Oncol 1999:3:976-83.

8 Balch CM, Buzaid AC, Atkins MB, et al. Final version of the American joint committee on cancer staging system for cutaneous melanoma. J Clin Oncol 2001;19:3635-48.

9 White RR, Stanley WE, Johnson JL, et al. Long-term survival in 2,505 patients with melanoma with regional lymph node metastasis. Ann Surg 2002;235:879-87.

10 McCready DR, Ghazarian DM, Hershkop MS, et al. Sentinel lymph-node biopsy after previous wide local excision for melanoma. Can J Surg 2001:44:432-4.

11 Cochran AJ, Huang RR, Guo J, et al. Current practice and future directions in pathology and laboratory evaluation of the sentinel node. Ann Surg Oncol 2001;8(9S): 13-17.

12 Jansen L, Nieweg OE, Peterse JL, et al. Reliability of sentinel lymph node biopsy for staging melanoma. Br J Surg 2000:87:484-9.

13 Gershenwald JE, Colome MI, Lee JE, et al. Patterns of recurrence following a negative sentinel lymph node biopsy in 243 patients with stage I or II melanoma. J Clin Oncol 1998;16:2253-60.

14 Morton DL, Thompson JF, Essner R, et al. Validation of the accuracy of intraoperative lymphatic mapping and sentinel lymphadenectomy for earlystage melanoma: a multicentre trial-multicenter selective lymphadenectomy trial group. Ann Surg 1999;230:453-63.

15 McMasters KM, Reintgen DS, Ross MI, et al. Sentinel lymph node biopsy for melanoma: controversy despite widespread agreement. J Clin Oncol $2001 ; 19: 2851-5$.
16 Muller MG, Van Leeuwen PA, de Lang-de Klerk ES, et al. The sentinel lymph node status is an important factor for predicting clinical outcomes in patients with stage 1 or 2 cutaneous melanoma. Cancer 2001;91:2401-8.

17 Russell-Jones R, Acland K. Sentinel node biopsy in the management of malignant melanoma. Clin Exp Dermatol 2001;26:463-8.

18 Gadd MA, Cosimi AB, Yu J, et al. Outcome of patients with melanoma and histologically negative sentinel lymph nodes. Arch Surg 1999;134:381-7.

19 Treseler PA, Tauchi PS. Pathologic analysis of the sentinel lymph node. Surg Clin North Am 2000;80:1695-719.

20 Goscin C, Glass LF, Messina JL. Pathologic examination of the sentinel lymph node in melanoma. Surg Oncol Clin North Am 1999;8:427-34

21 Messina JL, Glass LF, Cruse CW, et al. Pathologic examination of the sentinel lymph node in malignant melanoma. Am J Surg Pathol 1999;23:686-90.

22 Morton DL, Wen DR, Foshag $\amalg$, et al. Intraoperative lymphatic mapping and selective cervical lymphadenectomy for early-stage melanomas of the head and neck. J Clin Oncol 1993;11:1751-6.

23 Yu LL, Flotte TJ, Tanabe KK, et al. Detection of microscopic melanoma metastases in sentinel lymph nodes. Cancer 1999:86:617-27.

24 Uren RF, Thompson JF, Howman-Giles R. Sentinel lymph node biopsy in patients with melanoma and breast cancer. Intern Med J 2001;31:547-53.

25 Starz H, Balda BR, Kramer KU, et al. A micromorphometry-based concept for routine classification of sentinel lymph node metastases and its clinical relevance for patients with melanoma. Cancer 2001;91:2110-21.

26 Belhocine T, Pierard G, De Labrassinne M, et al. Staging of regional nodes in AJCC stage I and II melanoma: 18FDG PET imaging versus sentinel node detection. Oncologist 2002;7:271-8.

27 Chu KU, Turner RR, Hansen NM, et al. Do all patients with sentinel node metastasis from breast carcinoma need complete axillary node dissection? Ann Surg 1999;229:536-41.

28 Czerniecki BJ, Scheff AM, Callans LS, et al. Immunohistochemistry with pancytokeratins improves the sensitivity of sentinel lymph node biopsy in patients with breast carcinoma. Cancer 1999;85:1098-103.

29 Wagner JD, Davidson D, Coleman JJ, et al. Lymph node tumour volumes in patients undergoing sentinel lymph node biopsy for cutaneous melanoma. Ann Surg Oncol 1999;6:398-404.

30 Bahemer FA, Hantirah S, Baum H-P. Rapid and unbiased estimation of the volume of cutaneous malignant melanoma using Cavalieri's principle. Am J Dermatopathol 1996;18:159-64.

31 Cochran AJ, Lana AMA, Wen D-R. Histomorphometry in the assessment of prognosis in stage II malignant melanoma. Am J Surg Pathol 1989;13:600-4.

32 Thelmo MC, Sagebiel RW, Treseler PA, et al. Evaluation of sentinel lymph node status in spindle cell melanomas. J Am Acad Dermatol 2001;44:451-5.

33 Ascierto P, Mozzillo N, Caraco C, et al. Epithelioid cell-type melanoma as a prognostic factor of poor response to immunological treatment. Ann Oncol 2000;11:1504.

34 Ranieri JM, Wagner JD, Azuaje R, et al. Prognostic importance of lymph node tumor burden in melanoma patients staged by sentinel node biopsy. Ann Surg Oncol 2002;9:975-81. 